\title{
Clinical implications of neuropsychiatric systemic lupus erythematosus
}

\author{
Anselm Mak, Roger Chun Man Ho \& Chak Sing Lau
}

\begin{abstract}
SUMMARY
Systemic lupus erythematosus (SLE) is a disorder which can affect the central nervous system and result in a broad range of psychiatric syndromes such as psychosis, mood disorders, acute confusion and cognitive dysfunction. Despite the robust nomenclature of neuropsychiatric SLE (NPSLE), psychiatric syndromes in patients are often non-specific and may be secondary to concurrent non-SLE-related conditions and complications of medical therapies. Although the exact immunopathological mechanism for psychiatric presentation remains elusive, prompt exclusion of other factors contributing to the psychiatric symptoms coupled with effective assessment strategies and management with immunosuppression and psychiatric therapy are imperative. Psychiatrists and rheumatologists must work in close liaison to identify, treat and prognosticate patients with psychiatric syndromes in order to improve their quality of life, vocational aptitude and, ultimately, survival.
\end{abstract}

\section{DECLARATION OF INTEREST}

None.

Systemic lupus erythematosus (SLE) is an autoimmune disorder mediated by the immunecomplex and characterised by its protean clinical manifestation and multisystemic involvement (Mak 2007a). Neuropsychiatric manifestation of SLE (NPSLE) is one of the major and most damaging presentations. It comprises a wide range of neurological syndromes affecting the central, peripheral and autonomic nervous systems, as well as psychiatric syndromes. In view of the diverse clinical manifestation of NPSLE, the American College of Rheumatology research committee devised a nomenclature which gives case definitions for 19 neuropsychiatric syndromes in SLE (ACR Ad Hoc Committee on Neuropsychiatric Lupus Nomenclature 1999) (Box 1). Albeit comprehensive, none of the syndromes described are specific to SLE and, in fact, non-SLE causes such as complications of lupus therapy, infection of the central nervous system (CNS), metabolic dysfunction and drug intoxication may contribute to them. Hence, these complications must be excluded before being ascribed to the underlying immunopathogenic mechanisms (Mak 2007b).

Although overt psychiatric symptoms are often clinically obvious, subtle changes such as mild cognitive impairment are often left unnoticed. This underscores the need for careful and robust clinical assessment tools which help identify these clinically important psychopathologies. In addition, how psychiatrists formally diagnose and evaluate a wide variety of lupus-related psychiatric syndromes and their impacts by using validated assessment instruments and ultimately manage these syndromes in conjunction with rheumatologists needs to be addressed.

This article reviews the immunopathogenesis, clinical manifestation and therapeutic options for NPSLE, and highlights the indispensable role of psychiatrists working with rheumatologists in the collaborative management of these conditions.

\section{ARTICLE}

Anselm Mak is Assistant Professor of Medicine and an associate consultant rheumatologist in the Department of Medicine, Yong Loo Lin School of Medicine, National University of Singapore. His research interest is lupus-related damage, especially NPSLE-related damage, and lupus nephritis.

Roger Chun Man Ho is Assistant Professor of psychiatry and a specialist psychiatric registrar at the National University of Singapore. He has a special interest in psychoneuroimmunology. Chak Sing Lau is Professor of Medicine and a consultant rheumatologist at the Ninewells Hospital and Medical School, University of Dundee, Scotland. His research interests include immunogenetics, $T$ and $B$ cell functions, and apoptosis in SLE.

Correspondence DrRoger Chun Man Ho, Department of Psychological Medicine, National University Hospital, 5 Lower Kent Ridge Road, Singapore 110974. Email: pcmrhcm@nus.edu.sg

BOX 1 Neuropsychiatric manifestations of SLE ${ }^{a}$ and prevalence rates ${ }^{b}$

$\begin{array}{ll}\text { Central nervous system } & \text { Peripheral nervous system } \\ \text { - Aseptic meningitis } & \text { - Acute inflammatory demyelinating } \\ \text { - Cerebrovascular disease } & \text { polyradiculoneuropathy (Guillain-Barré } \\ \text { - Demyelinating syndrome } & \text { syndrome) } \\ \text { - Headache (including migraine and benign } & \text { - Autonomic disorder } \\ \text { intracranial hypertension) } & \text { - Mononeuropathy (single/multiplex) } \\ \text { - Movement disorder (chorea) } & \text { - Myasthenia gravis } \\ \text { - Myelopathy } & \text { - Cranial neuropathy } \\ \text { - Seizure disorders } & \text { - Plexopathy } \\ \text { - Acute confusional state (<1\%) } & \text { - Polyneuropathy } \\ \text { - Anxiety disorder } & \\ \text { - Cognitive dysfunction (55-80\%) } & \\ \text { - Mood disorder (14-57\%) } & \\ \text { - Psychosis (0-8\%) } & \\ \text { a. ACR Ad Hoc Committee on Neuropsychiatric Lupus Nomenclature (1999) } \\ \text { b. Hanly (2005) }\end{array}$

Central nervous system

- Aseptic meningitis

- Cerebrovascular disease

- Demyelinating syndrome

- Headache (including migraine and benign intracranial hypertension)

- Movement disorder (chorea)

- Myelopathy 


\section{Epidemiology, pathogenesis and therapeutic options}

\section{Epidemiology}

Neuropsychiatric manifestations in people with SLE are fairly common, with a prevalence of $17-75 \%$. The wide range reflects possible interethnic variations of lupus manifestation and different criteria for diagnosis (Stojanovich 2007). Neuropsychiatric manifestations of SLE are complex:

- they can occur at any time during the course of the disorder or even precede its onset

- they can occur during the active or quiescent phase of lupus

- patients may present with single or multiple neurological and/or psychiatric manifestations (Hanly 2005).

\section{Pathogenesis}

Regarding the nomenclature of NPSLE, clinical manifestation is divided into diffuse and focal syndromes, largely depending on the anatomical sites of the CNS pathology (Fig. 1). Several immunopathogenic mechanisms giving rise to NPSLE syndromes have been proposed. Although NPSLE syndromes such as stroke and peripheral neuropathy are likely to be related to thromboembolism and vasculopathy, the mechanisms for diffuse psychiatric syndromes such as depression and psychosis are related to auto-antibody-mediated neurotoxicity. Among
BOX 2 Auto-antibodies involved in NPSLE

- Anti-ribosomal P antibodies

- NMDA receptor antibodies

- Antiphospholipid antibodies

- A proliferation-inducing ligand (APRIL) for B cell survival and function (George-Chandy 2008)

- Antihistone antibodies (Sun 2008)

patients with lupus, anti-ribosomal $\mathrm{P}$ antibodies are higher in individuals who have depression and/or psychosis than in those who do not (Arnett 1996). Anti- $N$-methyl- $D$-aspartate (anti-NMDA) receptor antibodies have attracted substantial attention, as NMDA receptors are capable of binding to the neurotransmitter glutamate. The hippocampus expresses the highest number of NR2 NMDA receptors and studies have demonstrated that antagonising NMDA receptors can cause hallucinations and paranoia (Jentsch 1999). The exact reason why anti-NMDA receptor antibodies can lead to such diffuse NPSLE syndromes is not clear, although antagonisation of NR2 receptors has been shown to induce apoptosis of neurons and subsequent neuronal injury in a way reminiscent of excitatory amino-acid toxicity (Lipton 1994).

Antiphospholipid antibodies are associated with focal neurological manifestations such as cerebrovascular diseases (Petri 2007). Box 2 summarises the auto-antibodies involved in NPSLE.

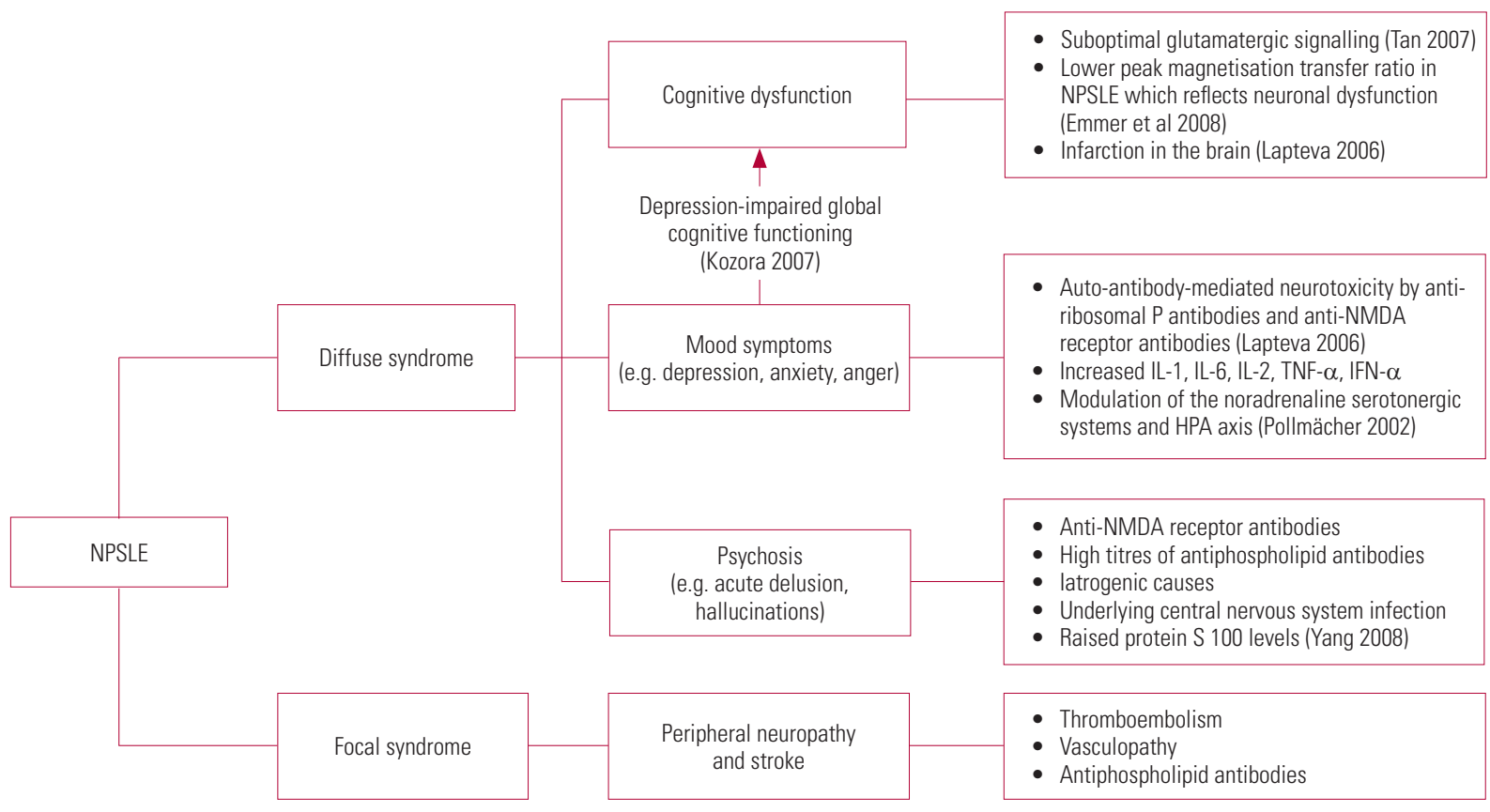


Several lines of evidence have demonstrated that serum pro-inflammatory cytokines are involved in depression in SLE. For example, patients with SLE and depression have elevated serum levels of interleukin (IL)-1, IL-6, IL-2, tumour necrosis factor (TNF)- $\alpha$ and interferon (IFN)- $\alpha$ (Maes 1995). Figure 2 summarises the roles of antibodies and cytokines in major neuropsychiatric symptoms and Box 3 lists the changes that occur.

\section{Therapeutic interventions}

Treatment options for NPSLE are very limited, as therapy mainly comprises high-dose corticosteroids plus an immunosuppressive agent as an induction, followed by switching to a less toxic immunosuppressive agent and lower dose of corticosteroid as maintenance.

Only one small randomised controlled trial has been published comparing intravenous methylprednisolone and intravenous cyclophosphamide as treatments for NPSLE (Baca 1999). The study found that significantly more patients responded to treatment in the cyclophosphamide group compared with the methylprednisolone group.

Apart from immunosuppressive therapies for NPSLE, auxiliary treatments such as antipsychotic, anti-anxiolytic and anti-epileptic agents are often required for controlling symptoms such as psychosis, anxiety and seizures.

\section{Cognitive impairment and lupus}

Cognitive impairment is one of the most common NPSLE syndromes, occurring in $20-80 \%$ of patients with lupus (Hanly 2005). The common cognitive abnormalities associated with NPSLE and their expected findings in neuropsychological tests are summarised in Table 1.

\section{Assessment}

As yet, no single bedside screening test is available, as the Mini-Mental State Examination (MMSE) was shown to be unhelpful in assessing neuropsychiatric manifestations in patients with SLE (Hanly 2005). Poole et al (2006) found that cognitive deficits were better assessed by performance-based tests of disability rather than a self-report.

\section{B0X 3 Diffuse changes in SLE \\ - Reduction in cerebral blood flow (Yoshida 2007) \\ - Loss of tissue integrity in brain parenchyma (Welsh 2007) \\ - Demyelination in white and grey matter (Huges 2007)}



FIG 2 Neuroanatomical locations and neuropsychiatric symptoms of SLE. HPA, hypothalamicpituitary-adrenal; NMDA, $N$-methyl- $D$-aspartate; NPSLE, neuropsychiatric systemic lupus erythematosus.

\section{Brain imaging}

Similar to schizophrenia, executive dysfunctions such as difficulty in multi-tasking, organisation and planning are common in NPSLE (Hanly 2005). In contrast to schizophrenia, the understanding of cognitive dysfunction did not evolve from the traditional 'lesion-based' approach, as earlier magnetic resonance imaging (MRI) findings were either non-specific or negative.

Diffusion tensor imaging has revealed early changes in the frontal lobe, genu corpus callosum and anterior internal capsule of patients with NPSLE (Zhang 2007). Monastero et al (2001) found that changes in psychiatric comorbidity such

\section{TABLE 1 Common cognitive abnormalities associated with neuropsychiatric systemic} lupus erythematosus and expected findings in neuropsychological tests

\begin{tabular}{|ll}
\hline Cognitive abnormalities & Expected findings in neuropsychological tests \\
\hline $\begin{array}{l}\text { Impairment in immediate, delayed, } \\
\text { retrieval and global memory }\end{array}$ & $\begin{array}{l}\text { Rey AVLT (trial I): problems with immediate word span; } \\
\text { difficulties with immediate recall trial after five oral } \\
\text { presentations of a 15-word list } \\
\text { Rey AVLT (trial VII): problems with retrieval efficiency; } \\
\text { delayed recall trial after } 30 \text { min }\end{array}$ \\
\hline $\begin{array}{ll}\text { Impairment in visuospatial activities } \\
\text { (e.g. affecting visual construction) }\end{array}$ & $\begin{array}{l}\text { RCFT (copy phase): problems with constructional and } \\
\text { organisational ability; failure to copy a complex figure } \\
\text { WAIS (block design): failure to use blocks to construct }\end{array}$ \\
& $\begin{array}{l}\text { replicas of designs } \\
\text { RCFT (immediate recall phase): problems with visuospatial } \\
\text { memory; failure to recall drawing after } 3 \text { min }\end{array}$ \\
\hline Poor attention and concentration & $\begin{array}{l}\text { WAIS (digit span): failure to repeat random number } \\
\text { sequences forwards and backwards }\end{array}$ \\
\hline Difficulties with abstract thinking & $\begin{array}{l}\text { Providing superficial explanation when asked to interpret } \\
\text { proverbs }\end{array}$ \\
\hline Reduction in psychomotor speed & $\begin{array}{l}\text { WAIS (digit symbol): failure to complete the task of filling } \\
\text { the blank spaces with symbols paired with numbers in 90s }\end{array}$ \\
\hline
\end{tabular}

AVLT, Auditory Verbal Learning Test; RCFT, Rey Complex Figure Test; WAIS, Wechsler Adult Intelligence Scale. a. From Tektonidou (2006) 
as depressive disorder parallel changes in cognitive function. Hence, psychotropic medication such as antidepressants may improve both mood status and cognitive function. The effect of cholinesterase inhibitors in individuals with lupus and cognitive impairment is not known.

\section{Cognitive rehabilitation}

Cognitive rehabilitation should also be considered, as it was shown to be promising in patients with schizophrenia and cognitive dysfunction (Wykes 1999). Since cognitive impairment pertaining to NPSLE is heterogeneous, one approach to cognitive rehabilitation is to focus on subgroups of patients with specific cognitive abnormalities (e.g. of memory and attention). Larger, controlled trials of cognitive rehabilitation are required to identify specific and effective treatment strategies, long-term outcomes and cost-effectiveness of treatment.

\section{Psychosis and lupus}

Appenzeller et al (2008) conducted a longitudinal study of patients with SLE for 9 years and identified primary psychotic disorder in $17 \%$ of the cohort. Of the patients presenting with primary psychotic disorder, $66 \%$ of cases were related to NPSLE, $31 \%$ were associated with corticosteroids and $3 \%$ were related to neither NPSLE or medication. Psychosis secondary to SLE at disease onset occurred in $21 \%$ of patients presenting with primary psychotic disorder and $45 \%$ of cases were associated with positive antiphospholipid antibodies.

\section{Psychotic symptoms in SLE}

Case study: Corticosteroid-induced psychosis

A 19-year-old woman was diagnosed with SLE when she presented with arthralgia, malar rash and subsequent diffuse progressive glomerulonephritis. Aggressive immunosuppression comprising intravenous pulse methylprednisolone followed by oral prednisolone and cyclophosphamide was given.
Although her lupus activity was under control, she started to suffer from insomnia and mania. She accused somebody of following and harming her. She had no visual hallucinations, and neurological examination revealed no focal deficit. Thorough metabolic and infection screening was negative and no abnormalities were shown on brain MRI. The diagnosis of corticosteroid-induced psychosis was made and a subsequent prednisolone taper was accompanied by a progressive improvement of her psychotic symptoms over the next 3 weeks. Adjunctive antipsychotics were titrated to zero over a further 2 months.

Cases similar to that of the young woman described above are often referred to psychiatrists. In referrals of young people with a first episode of psychosis it is important to rule out physical disorders such as SLE (Fernandez 2007). The main clinical features of psychosis in NPSLE are acute delusions and hallucinations. As the typical age at presentation of NPSLE-related psychosis is in the early 20s, it is often difficult to differentiate it from schizophreniform psychosis. Corticosteroids also contribute to psychosis through ischaemia and subsequent hippocampal damage (Wolkowitz 1997). Table 2 summarises the differences between psychosis related to NPSLE and to corticosteroids.

\section{Antipsychotics and their side-effects}

Antipsychotic medication commonly used in schizophrenia can also be given to patients with NPSLE. Lupus-related bodily changes often affect the pharmacokinetics and pharmacodynamics of antipsychotic drugs, leading to side-effects that can be persistent and disabling. Chlorpromazine, for example, is well known to cause drug-induced lupus. It may aggravate photosensitivity and complicate the existing manifestations of lupus (Harth 1996). Although recent studies have indicated that the new atypicals may have a more favourable side-effect profile, agranulocytosis from atypical antipsychotics such as olanzapine has been reported ( $\mathrm{Su} 2007)$.

Comparison between neuropsychiatric systemic lupus erythematosus (NPSLE) psychosis and corticosteroid-induced psychosis (Appenzeller 2008)

\begin{tabular}{|c|c|c|c|c|}
\hline & Onset & Risk factors & Resolution & $\begin{array}{l}\text { Predictors of } \\
\text { recurrence }\end{array}$ \\
\hline $\begin{array}{l}\text { NPSLE } \\
\text { psychosis }\end{array}$ & $\begin{array}{l}\text { Acute } \\
\text { Mean age at onset: } 25 \text { years }\end{array}$ & $\begin{array}{l}\text { High disease activity } \\
\text { Other NPSLE manifestations such as } \\
\text { depression or cognitive impairment } \\
\text { Absence of cutaneous manifestations } \\
\text { such as malar rash and photosensitivity }\end{array}$ & $\begin{array}{l}\text { May require antipsychotic } \\
\text { treatment }\end{array}$ & $\begin{array}{l}\text { Positive } \\
\text { antiphospholipid } \\
\text { antibodies in } \\
\text { moderate to high } \\
\text { titres }\end{array}$ \\
\hline $\begin{array}{l}\text { Corticosteroid- } \\
\text { induced } \\
\text { psychosis }\end{array}$ & $\begin{array}{l}\text { New onset of psychosis that } \\
\text { appears temporally within } 8 \text { weeks } \\
\text { of being admitted to hospital or } \\
\text { augmentation of steroids }\end{array}$ & Hypoalbuminemia & $\begin{array}{l}\text { Psychosis will be resolved } \\
\text { completely after reduction of } \\
\text { steroid dosage without additional } \\
\text { immunosuppressive agents }\end{array}$ & $\begin{array}{l}\text { Increments of } \\
\text { corticosteroid to } \\
\text { control systemic } \\
\text { manifestations }\end{array}$ \\
\hline
\end{tabular}


Research addressing the use of first-generation antipsychotics in people with lupus and psychosis is sparse. These drugs are not recommended because they may exacerbate NPSLE-associated movement disorders (Nishimura 2003).

No data are yet available from controlled trials regarding the efficacy and safety of secondgeneration antipsychotics. As stroke is one of the NPSLE syndromes, risperidone and olanzapine should be avoided. These drugs are a known risk factor for cerebrovascular adverse events in elderly patients without SLE (Herrmann 2005). Quetiapine and sulpiride are recommended, as there are no reports of adverse effects in individuals with NPSLE.

In severe NPSLE, pulsed intravenous cyclophosphamide has produced significant improvement in psychotic symptoms (Bodani 2003). The medication was well tolerated in adult and paediatric patients.

\section{Non-pharmacological interventions}

Research into non-pharmacological treatment for NPSLE-associated psychosis is limited. Although there is compelling evidence to support the use of cognitive-behavioural therapy (CBT) in modifying delusional beliefs and controlling hallucinations in people with schizophrenia (Garety 1994; Kuipers 1997), no study has assessed the effect of CBT on patients with NPSLE-associated psychosis; however, the same principles can be applied.

Clinicians should address the nature of untreated SLE-related psychosis and its negative impact on both patient and caregivers if not treated. Psychoeducation is often useful to enhance insight and understanding.

\section{Depression, anxiety and lupus}

Depression and anxiety are the most frequently encountered psychiatric problems in patients with lupus (Wekking 1993), but there are no specific features that are unique to SLE (Hanly 2005). The use of different criteria for diagnosis has resulted in a wide prevalence (24-57\%) across studies (Brey 2002). For practical purposes, it is probably most helpful to consider depression and anxiety as dimensions rather than categories (Goldberg 1992). ${ }^{\dagger}$ Depression and anxiety in SLE are often accompanied by other problems, such as poor anger control.

In SLE, depression that presents with predominantly somatic complaints (e.g. pain and lethargy) may remain undiagnosed as these symptoms overlap those of lupus itself. For medical patients such as those with SLE, the Hamilton Rating Scale for Depression (HRSD) has low sensitivity in detecting depression as its questions are too direct (e.g. have you had suicidal thought in the past 3 weeks?) and are intended for psychiatric patients with clear-cut depressive disorder. Most medical patients will get a very low score on the HRSD but this does not mean they are not depressed or have good mental health. Stoll et al (2001) therefore proposed the routine use of the Short Form (SF)-36 to detect depression when assessing patients with SLE. The SF-36 mental health score closely correlates with the severity of depression on the HRSD but its questions are more relevant to the everyday life of people with SLE (e.g. have your physical health or emotional problems interfered with activities like visiting relatives?).

\section{Antidepressants}

Data regarding the use of antidepressants in individuals with SLE stem from just a few case reports. Selective serotonin reuptake inhibitors (SSRIs) can be useful in treating depression in NPSLE, and psychiatrists need to be aware of specific side-effects of different antidepressants. Cassis et al (2005) and Hill et al (1998) have reported erythematosus skin lesions related to bupropion and sertraline use. Fluoxetine can cause extrapyramidal side-effects (Fallon 1991).

Douglas and colleagues (1982) reported a case of depression caused by lupus cerebritis that was successfully treated by electroconvulsive therapy (ECT). With the development of a variety of antidepressants, the use of ECT to treat depression in people with SLE has become second-line in clinical practice. Nevertheless, ECT does play a role in treating manic psychosis and catatonia stemming from NPSLE (Ditmore 1992).

The role of psychological therapies and nonpharmacological strategies such as CBT, cognitive remediation relaxation therapy, sleep hygiene and regular exercise should also be considered.

\section{Fatigue and lupus}

Fatigue has been reported as one of the most common and most disabling symptoms in SLE, with a prevalence of up to 80\% (Krupp 1990). Eleven per cent of patients in the UK fulfilled the American College of Rheumatology classification criteria for fibromyalgia (Taylor 2000), a condition characterised by chronic fatigue syndrome for more than 3 months and multiple tender spots.

\section{Case study: Fibromyalgia in SLE}

A 50-year-old housewife with long-standing SLE has been treated with prednisolone and azathioprine for the past 3 years. She started to complain of easy fatigue, poor sleep and generalised body aches and pains 6 months ago. In addition, she noted
${ }^{\dagger}$ A simplification of the current classifications of mental disorder will be discussed by David Goldberg in the January 2010 issue of Advances. Ed. 
BOX 4 Factors contributing to fatigue in SLE

- Insomnia and high bodily pain

- Depression

- Anxiety

- Loss of muscular power

- Low levels of aerobic activity

- Associated fibromyalgia

(Tench 2000)

having a dry mouth and dysuria; no other systemic symptoms were observed. Physical examination revealed multiple tender spots over the neck and trunk. Thorough investigation showed no evidence of arthritis and her urine was sterile. Serology revealed no evidence of active lupus and metabolic screening did not suggest electrolyte imbalance or endocrinopathy. She was referred by her rheumatologist to a psychiatrist and the diagnosis of fibromyalgia was made. Eight weeks after treatment with fluoxetine, the symptoms of fibromyalgia substantially improved.

Box 4 summarises the key factors that contribute to fatigue in SLE. Tench et al (2000) have suggested that myalgia and arthralgia related to lupus may disturb sleep, resulting in daytime fatigue. Increased levels of anxiety and depression are also associated with increased SLE-related fatigue (Ward 2002).

\section{Clinical assessment}

Symptoms of fatigue can be rated using the Fatigue Severity Score developed by Krupp et al (1990). It is also worth considering laboratory tests to rule out underlying medical conditions such as anaemia, hypothyroidism and malignancies.

B0X 5 The psychiatrist's role in the management of NPSLE

The psychiatrist can help the general practitioner and the rheumatology team by:

- assessing the reasons for referral, precipitating factors and potential risk

- empathetic listening and eliciting an emotional response from the patient regarding their view of the illness, threat to self-image, perceived control over the illness, and prognosis

- obtaining a collateral history from the general practitioner and family as the patient may be cognitively impaired

- linking the pathogenesis of NPSLE and psychiatric symptoms
- determining the role of psychosocial factors in the causation and maintenance of psychiatric symptoms

- explaining the psychiatric formulation and decisions to other specialists, paramedical staff, patient and family

- advising on the pharmacological and psychological management of psychiatric symptoms

- coordinating psychiatric services such as cognitive remediation

- training practice nurses and general practitioners to provide psychological interventions in the primary care or community setting

\section{Treatment}

Treatment of fatigue in individuals with SLE is mainly symptomatic. Concomitant depressive disorder and sleep disturbance which may lead to fatigue should be identified and treated (McKinley 1995). An SSRI such as fluoxetine may improve mood and energy levels.

Psychological interventions such as lifestyle changes, including sleep hygiene, relaxation and regular exercise programmes, are safe and helpful. Psychological interventions such as lifestyle changes, including sleep hygiene, relaxation and regular exercise programmes, are safe and helpful. Tench et al (2003) conducted a randomised controlled trial in which patients with SLE were assigned to a 12 -week programme of aerobic exercise, relaxation therapy or no intervention. At the end of the 12 weeks they compared the participants' physiological, symptomatic and functional changes. Graded exercise led to significantly greater overall improvement than relaxation therapy or no intervention.

\section{The role of the psychiatrist}

In- and out-patient referrals are the usual routes by which rheumatologists obtain psychiatric opinions on patients with NPSLE, but they have insufficient face-to-face communication with psychiatrists. In the consultation-liaison model of practice, however, psychiatrists attend ward rounds and discuss the diagnosis and management plan with the rheumatology team. The advantages of this include better communication, which allows the psychiatrist a broader view, clarifies uncertainties and leads to agreement on the diagnosis. It may involve a longitudinal follow-up to address difficult issues and review responses to various treatment strategies. The psychiatrist needs to formulate a framework for assessing patients with NPSLE. The psychiatrist's role in the management of NPSLE is summarised in Box 5.

\section{Conclusions}

Psychiatric symptoms often occur in patients with SLE. Although the American College of Rheumatology nomenclature for NPSLE is comprehensive, psychiatric symptoms described in individuals with SLE are neither sensitive nor specific. Prompt recognition of psychiatric symptoms, with exclusion of aetiologies such as infection, metabolic or endocrine dysfunction and drug intoxication, is absolutely necessary.

Further research is required to explore the possibility of using anti-neuronal antibodies, electroneurophysiological studies and diffusion 
tensor imaging in diagnosing NPSLE, and to assess the efficacy of psychotropic medication and psychotherapy.

Close liaison between psychiatrists and rheumatologists undoubtedly facilitates effective assessment and management of the disease, which can improve the quality of life of individuals with SLE and associated psychiatric syndromes.

\section{References}

ACR Ad Hoc Committee on Neuropsychiatric Lupus Nomenclature (1999) The American College of Rheumatology nomenclature and case definitions for neuropsychiatric lupus syndromes. Arthritis and Rheumatism; 42 : 599-608.

Appenzeller S, Cendes F, Castallat L (2008) Acute psychosis in systemic lupus erythematosus. Rheumatology International; 28: 237-43.

Arnett FC, Reveille JD, Moutsopoulos HM, et al (1996) Ribosomal P autoantibodies in systemic lupus erythematosus. Frequencies in different ethnic groups and clinical and immunogenetic associations. Arthritis and Rheumatism; 39: 1833-9.

Baca V, Lavalle C, García R, et al (1999) Favorable response to intravenous methylprednisolone and cyclophosphamide in children with severe neuropsychiatric lupus. Journal of Rheumatology; 26: 432-9

Bodani M, Kopelman MD (2003) A psychiatric perspective on the therapy of psychosis in systemic lupus erythematosus. Lupus; 12: 947-9.

Brey RL, Holliday SL, Saklad AR, et al (2002) Neuropsychiatric syndromes in lupus: prevalence using standardized definitions. Neurology; 58 : 1214-20.

Cassis TB, Callen JP (2005) Bupropion-induced subacute cutaneous lupus erythematosus. Australasian Journal of Dermatology; 46: 266-9.

Ditmore BG, Malek-Ahmadi P, Millis DM, et al (1992) Manic psychosis and catatonia stemming from systemic lupus erythematosus: response to ECT. Convulsive Therapy; 8: 33-7.

Douglas CJ, Schwartz HI (1982) ECT for depression caused by lupus cerebritis: a case report. American Journal of Psychiatry; 139: 1631-2.

Driver CB, Wallace DJ, Lee JC, et al (2008) Clinical validation of the watershed sign as a marker for neuropsychiatric systemic lupus erythematosus. Arthritis and Rheumatism (Arthritis Care \& Research); 59: 332-7.

Emmer BJ, Steup-Beekman GM, Steens SC, et al (2008) Correlation of magnetization transfer ratio histogram parameters with neuropsychiatric systemic lupus erythematosus criteria and proton magnetic resonance spectroscopy: association of magnetization transfer ratio peak height with neuronal and cognitive dysfunction. Arthritis and Rheumatism; 58 : $1451-7$

Fallon BA, Liebowitz MR (1991) Fluoxetine and extrapyramidal symptoms in CNS lupus. Journal of Clinical Psychopharmacology; 11: 147-8.

Fernandez G, Gorriti M, Garcia-Vicuna R, et al (2007) Psychosis leading to the diagnosis of unrecognized systemic lupus erythematosus: a case report. Rheumatology International; 27: 883-5.

Garety PA, Kuipers L, Fowler D, et al (1994) Cognitive behavioural therapy for drug resistant psychosis. British Journal of Medical Psychology; 67: 259-71.

George-Chandy A, Trysberg E, Eriksson K (2008) Raised intrathecal levels of APRIL and BAFF in patients with systemic lupus erythematosus: relationship to neuropsychiatric symptoms. Arthritis Research and Therapy; 10: R97.

Goldberg D, Huxley P (1992) Common Mental Disorders. Routledge.

Harth Y, Rapoport M (1996) Photosensitivity associated with antipsychotics, antidepressants and anxiolytics. Drug Safety; 14: 252-9.

Hanly JG, Harrison MJ (2005) Management of neuropsychiatric lupus. Best Practice and Research Clinical Rheumatology; 19: 799-821.

Herrmann N, Lanctôt KL (2005) Do atypical antipsychotics cause stroke? Central Nervous System Drugs; 19: 91-103.
Hill VA, Hepburn NC (1998) Sertraline associated discoid lupus erythematosus-like eruption. Journal of the Royal Army Medical Corps; 144: $109-10$

Hughes M, Sundgren PC, Fan X, et al (2007) Diffusion tensor imaging in patients with acute onset of neuropsychiatric systemic lupus erythematosus: a prospective study of apparent diffusion coefficient, fractional anisotropy values, and eigen values in different regions of the brain. Acta Radiologica; 48: 213-22.

Jentsch JD, Roth RH (1999) The neuropsychopharmacology of phencyclidine: from NMDA receptor hypofunction to the dopamine hypothesis of schizophrenia. Neuropsychopharmacology; 20: 201-25.

Kozora E, Arciniegas D, Zhang L, et al (2007) Neuropsychological patterns in systemic lupus erythematosus patients with depression. Arthritis Research and Therapy; 9: R48.

Krupp LB, LaRocca NG. Muir J, et al (1990) A study of fatigue in systemic lupus erythematosus. Journal of Rheumatology; 17: 1450-2.

Kuipers E, Garety P, Fowler D, et al (1997) London-East Anglia randomised controlled trial of cognitive-behavioural therapy for psychosis. I: effects of treatment phase. British Journal of Psychiatry; 171: 319-27.

Lapteva L, Nowak M, Yarboro CH, et al (2006) Anti-N-methyl-D-aspartate receptor antibodies, cognitive dysfunction, and depression in systematic lupus erythematosus. Arthritis and Rheumatism; 54: 2505-14.

Lipton SA, Rosenberg PA (1994) Excitatory amino acids as a final common pathway for neurologic disorders. New England Journal of Medicine; 330: 613-22.

Mak A, Mok CC, Chu WP, et al (2007a) Renal damage in systemic lupus erythematosus: a comparative analysis of different age groups. Lupus; 16: $28-34$.

Mak A, Chan BP, Yeh IB, et al (2007b) Neuropsychiatric lupus and reversible posterior leucoencephalopathy syndrome: a challenging clinical dilemma. Rheumatology; 47: 256-62

Maes M, Meltzer HY, Bosmans E, et al (1995) Increased plasma concentrations of interleukin-6, soluble interleukin-6, soluble interleukin-2 and transferrin receptor in major depression. Journal of Affective Disorder; 34: $301-9$

McKinley P, Ouellette SC, Winkel G (1995) The contributions of disease activity, sleep patterns and depression to fatigue in systemic lupus erythematosus. Arthritis and Rheumatism; 38: 826-34.

Monastero R, Bettini P, Del Zotto E, et al (2001) Prevalence and pattern of cognitive impairment in systemic lupus erythematosus patients with and without overt neuropsychiatric manifestations. Journal of Neurological Sciences; 184: 33-9.

Nishimura K, Omori M, Horikawa N, et al (2003) Risperidone in the treatment of acute confusional state due to neuropsychiatric lupus erythematosus: case report. International Journal of Psychiatry in Medicine; 33: 299-303.

Petri M (2007) The Hopkins Lupus Pregnancy Center: ten key issues in management. Rheumatic Diseases Clinics of North America; 33: 227-35.

Pollmächer T, Haack M, Schuld A, et al (2002) Low levels of circulating inflammatory cytokines. Do they affect human brain functions? Brain, Behaviour, and Immunity; 16: 525-32.

Poole JL, Atanasoff G, Pelsor JC, et al (2006) Comparison of a self-report and performance-based test of disability in people with systemic lupus erythematosus. Disability and Rehabilitation; 28: 653-8.

Stojanovich L, Zandman-Goddard G, Pavlovich S, et al (2007) Psychiatric manifestations of systemic lupus erythematosus. Autoimmunity Review: 6. $421-6$.

Stoll T, Kauer Y, Buchi S, et al (2001) Prediction of depression in systemic lupus erythematosus patients using SF-36 Mental Health scores. Rheumatology; 40: 695-8.

Su JA, Wu CH, Tsang HY (2007) Olanzapine-induced agranulocytosis in systemic lupus erythematosus: a case report. General Hospital Psychiatry; 29: 75-7.

Sun XY, Shi J, Han L, et al (2008) Anti-histones antibodies in systemic lupus erythematosus: prevalence and frequency in neuropsychiatric lupus. Journal of Clinical Laboratory Analysis; 22: 271-7. 
Tan HY, Chen Q, Sust S, et al (2007) Epistasis between catechol-0methyltransferase and type II metabotropic glutamate receptor 3 genes on working memory brain function. Proceedings of the National Academy of Sciences of the United States of America; 104: 12536-41.

Taylor J, Skan J, Erb N, et al (2000) Lupus patients with fatigue: is there a link with fibromyalgia syndrome? Rheumatology; 39: 620-3.

Tench CM, McCurdie I, White PD, et al (2000) The prevalence and associations of fatigue in systemic lupus erythematosus. Rheumatology; 39: 1249-54

Tench CM, McCarthy J, McCurdie I, et al (2003) Fatigue in systemic lupus erythematosus: a randomized controlled trial of exercise. Rheumatology; 42: $1050-4$.

Tektonidou MG, Varsou N, Kotoulas G, et al (2006) Cognitive deficits in patients with antiphospholipid syndrome. Archives of Internal Medicine; 166: 2278-84

Ward MM, Marx AS, Barry NN (2002) Psychological distress and changes in the activity of systemic lupus erythematosus. Rheumatology; 41: 184-8.

Wekking EM (1993) Psychiatric symptoms in systemic lupus erythematosus: an update. Psychosomatic Medicine; 55: 219-28.
Welsh RC, Rahbar H, Foerster B, et al (2007) Brain diffusivity in patients with neuropsychiatric systemic lupus erythematosus with new acute neurological symptoms. Journal of Magnetic Resonance Imaging; 26 : $541-51$.

Wolkowitz OM, Reus VI, Canick J, et al (1997) Glucocorticoid medication, memory and steroid psychosis in medical illness. Annals of the New York Academy of Sciences; 823: 81-96.

Wykes T, Reeder C, Corner J, et al (1999) The effects of neurocognitive remediation on executive processing in patients with schizophrenia. Schizophrenia Bulletin; 25: 291-307.

Yang XY, Lin J, Lu XY, et al (2008) Expression of S100B protein levels in serum and cerebrospinal fluid with different forms of neuropsychiatric systemic lupus erythematosus. Clinical Rheumatology; 27: 353-7.

Yoshida A, Shishido F, Kato K, et al (2007) Evaluation of cerebral perfusion in patients with neuropsychiatric systemic lupus erythematosus using 123I-IMP SPECT. Annals of Nuclear Medicine; 21: 151-8.

Zhang L, Harrison M, Heier LA, et al (2007) Diffusion changes in patients with systemic lupus erythematosus. Magnetic Resonance Imaging; 25: 399-405.

\section{EMIs}

\section{Theme: Laboratory markers}

Which of the laboratory markers below is most strongly associated with the following NPSLE symptoms?

1 'I hear a voice outside my house. She always sings to me. When I go out and check, there is nobody around.'

2 A young female with SLE complains of low mood and is noted to have cognitive decline.

3 A 22-year-old woman presented with malar rash on her face. What test would you order as a screening test?

\section{Options}

a Anti-double stranded DNA

b Complement proteins

c Anti-NMDA antibody

d Anticardiolipin antibody

e Antinuclear antibody

f Antiphospholipid antibodies

\section{Theme: Neuropsychological tests}

A 22-year-old architecture student presents with 5 years' history of SLE. She has become increasingly forgetful and appears depressed.
Her main complaint is of poor attention. Her academic results have deteriorated as she has difficulty with drafting and designing new buildings.

4 She has difficulty recalling three items during the Mini Mental State Examination (MMSE). You are concerned with her memory. Which test would you use to assess her immediate, delayed, retrieval and global memory?

5 As an architecture student, she is failing her assignment in drafting and design. You are concerned with her visuospatial abilities. Which test would you use to assess her constructional and organisation abilities?

6 On the MMSE, she appears to be slow. You are concerned with psychomotor retardation. Which test would you use to assess her psychomotor speed?

Options

g Rey Auditory Verbal Learning Test

h Rey Complex Figure Test

i Serial 7, 3 stage command orientation

j Wechsler Adult Intelligence Scale (digital span)

k Wechsler Adult Intelligence Scale (digit symbol)

I Stroop test

\section{Theme: Management of NPSLE}

Which of the above actions is the most appropriate in the following scenarios?

7 The general practitioner insists that his patient is frankly psychotic and needs to be detained in hospital under the Mental Health Act.

8 A patient with SLE feels that their illness is hopeless and there will be no cure.

9 A 28-year-old patient with SLE complains of tiredness and low energy level. They are not keen to take additional medication.

Options

$\mathrm{m}$ Empathetic listening and eliciting emotional response from the patient

n Linking the pathogenesis of NPSLE and psychiatric symptoms, and offering explanation to other professionals

o To prescribe an antidepressant

$\mathrm{p}$ To prescribe a benzodiazepine

q Encouraging the patient to do exercise on a regular basis 